\title{
The Rationale for Transanal Total Mesorectal Excision
}

\author{
Roger W. Motson, M.S., F.R.C.S. ${ }^{1}$ Antonio Lacy, M.D., F.A.S.C.R.S. (Hons.) ${ }^{2}$ \\ 1 The ICENI Centre, Colchester University Hospital \& Anglia Ruskin University, Colchester, Essex, United Kingdom \\ 2 The Hospital Clinic \& University of Barcelona, Barcelona, Spain
}

$\mathrm{T}$ Total mesorectal excision (TME) has transformed the outcome of surgery for patients with rectal cancer since its inception more than 25 years ago. ${ }^{1}$ Subsequently, with the introduction of laparoscopic surgery into the management of colon and rectal cancer, there has rightly been a concern that the benefits of TME not be lost with the laparoscopic approach. This has largely been answered by the randomized trials, COST, COLOR, and CLASICC, although only CLASICC included patients with rectal cancer. ${ }^{2-4}$

Although laparoscopic rectal resection was first performed for more than 20 years, it has only been performed more frequently in the past 10 years and still accounts for less than $50 \%$ of all surgery for rectal cancer. This has, at least in part, been due to the technical issues of operating laparoscopically in the pelvis, which can present the surgeon with considerable difficulties in accurately staying in the TME plane. This article discusses how performing the distal TME dissection transanally may resolve these issues.

\section{LIMITATIONS OF LAPAROSCOPIC TME}

It is now widely accepted that the magnified views obtained with the laparoscope enable better visualization for the dissection of the inferior mesenteric artery and vein and for the mobilization of the proximal colon. The hypogastric nerves can be clearly seen as they enter the pelvis and remain in view as the retrorectal dissection progresses. However, when the mesorectum is bulky, when there is a midrectal tumor, and/or when the pelvis is narrow,

Financial Disclosures: None reported.

Correspondence: Roger W. Motson, M.S., F.R.C.S., The ICENI Centre, Colchester University Hospital, Colchester, Essex, CO4 5JL, UK. E-mail: rwmotson@aol.com

Dis Colon Rectum 2015; 58: 911-913

DOI: 10.1097/DCR.0000000000000422

(c) The ASCRS 2015 dissection becomes extremely difficult. Medial retraction of the mesorectum requires more force to provide a view of the plane of dissection and grasping the mesorectum may risk damage to the thin fascia enveloping the mesorectal fat. Furthermore, it can be difficult to maintain a view of the nerves on the pelvic sidewall increasing the risk of potential injury. Trying to avoid causing such an injury may result in the surgeon keeping his dissection away from the side wall, which then risks breaching the mesorectum in close proximity to the tumor. Even when these difficulties are overcome, the view for the dissection of the rectal muscle tube just above the anus may also be limited. All of these difficulties are more likely to be encountered in the male pelvis.

Although there have been considerable developments in laparoscopic instrumentation, particularly in optics and camera systems with high definition and, more recently, 3-dimensional imaging, the instruments to dissect deep in the pelvis have not changed by any major degree. Robotic instruments with the addition of a wrist may aid dissection, but some space is still needed to take advantage of this benefit. The challenge of dividing the rectal muscle tube to complete the resection of the rectum in a manner that is comparable to open surgery has yet to be achieved. In open surgery, a single firing of the stapling device completely divides the rectum in a transverse plane. Laparoscopically, even with staplers that can angulate, a truly transverse division or anteroposterior division at 90 degrees to the wall of the rectum is not achievable, but much it may appear to be so on the laparoscopic monitor. Furthermore, it is rare for the transection to be performed with a single firing of the stapling device, and more than 2 firings is associated with a greater risk of anastomotic leak. ${ }^{5}$

\section{TRANSANAL SURGERY}

A transanal approach to the rectum is not new. In 1983, Parks described handsewn coloanal anastomosis as an 
alternative to a difficult anastomosis deep in the pelvis. ${ }^{6}$ The advent of the circular stapler largely consigned this technique to history. Parks also pioneered intersphincteric dissection during proctectomy for IBD, thus preserving a normal pelvic floor, which, in turn, laid the way for the introduction of restorative proctectomy by means of an ileal pouch-anal anastomosis (IPAA).,8 Subsequently, Marks developed the transanal transabdominal technique, which also used intersphincteric dissection for the distal dissection. ${ }^{9}$ Transanal endoscopic microsurgery, developed by Buess for intrarectal resections, added improved visualization by means of magnifying binocular optics and also the development of instruments to work in this confined space. ${ }^{10}$

The concept of transanal total mesorectal excision (TaTME) has been built on these techniques with the intention to reliably improve the view and therefore the accuracy of the dissection for the most inaccessible part of the dissection. Before the first clinical case was performed, the concept had been studied in both porcine and cadaveric models to validate the technique and confirm that it was technically feasible. ${ }^{11-13}$ Central to the development of TaTME was the adaptation of the access devices developed for single-incision laparoscopy to transanal use, such as the Gelpoint-Path transanal platform (Applied Medical, Inc, Rancho Santa Margarita, CA). This and a number of alternative devices brought the benefits of an improved view with magnification for the surgeon and, in particular, for the assistant when performing the transanal dissection - something that both the Parks and Marks techniques lacked.

\section{TRANSANAL TME}

The transanal approach to the distal rectal dissection arguably deals effectively with the shortcomings of the laparoscopic approach described above. TaTME defines the distal resection margin more precisely and allows the surgeon to perform the deep pelvic dissection without the need for difficult retraction, even in the deep, narrow male pelvis or in patients who are obese. The initial division of the rectal muscle tube is at right angles to the rectal wall and gains entry into the space between the muscles of the pelvic floor and the mesorectum. This allows accurate dissection in the correct plane close to the mesorectal fascia. As dissection progresses upward, the space in which the surgeon is working is getting wider with an ever-improving view, rather than the narrower and worsening view when the surgeon dissects from above. The view for the anterior dissection between the rectum and prostate is also significantly improved.

If the colon and proximal rectum have already been mobilized, it is immediately apparent when the transanal dissection enters the peritoneal cavity.
In most cases, with the exception of a particularly bulky tumor or mesorectum, it is possible for the rectum to be delivered transanally before division of the proximal colon. This has the advantage of unequivocal certainty that the anastomosis will not be under any tension, and, without an abdominal incision, wound infection or subsequent herniation cannot occur. The technique of colorectal anastomosis is similar to the early days of circular stapling, before the use of a crossstapling device became the standard way to complete the rectal resection. Pursestring sutures bring both the cut end of the divided proximal colon and the cuff or remaining distal rectum into close contact with the shaft of the circular stapler before closure and firing of the device, thus avoiding any potential risk from intersecting staple lines.

When the rectal tumor is close to the anorectal junction, it may not be possible to make the initial distal rectal transection described above, because the line of division would be too close to the tumor. In these cases it is possible to perform an intersphincteric dissection starting at the dentate line by using an external retractor, such as the Lone Star (Aquilant Surgical) device, developing the dissection into the mesorectal space before the pursestring suture is inserted to close the rectum. It is understandable that there is concern that, during this phase of the dissection, there might be the opportunity for tumor cells to enter the mesorectal space and lead to local recurrence. Plainly, it is incumbent on the surgeon to avoid any contact between the dissecting instruments and the lower border of the tumor. A tumoricidal washout, such as povidone iodine solution, after the pursestring suture is placed is also appropriate. Experience to date in Barcelona has not resulted in a high incidence of local recurrence; in the initial follow-up of 140 patients, median 15 months, there have been 3 incidents of local recurrence (2.1\%), which is comparable to conventional open or laparoscopic TME. In addition, the long experience of Marks with the transanal transabdominal technique, in which the tumor is also in view initially, has not lead to an increased incidence of local recurrence. ${ }^{11}$

\section{TOP-DOWN FIRST, BOTTOM-UP FIRST, OR SYNCHRONOUS DISSECTION?}

For most surgeons considering this technique, it seems logical to perform the initial steps of the TME as they have already been doing - laparoscopic division of the inferior mesenteric vessels, mobilization of the left colon and splenic flexure, identification of the ureters and pelvic nerves, followed by dissection of the upper mesorectum until reaching a point where the dissection is becoming difficult. At this point, the surgeon would move to the perineum and 
dissect transanally to complete the mobilization. This approach avoids pneumodissection of the retroperitoneum and mesentery, which occurs with the initial dissection from below and may distort the anatomy. The top-down approach also avoids the possibility of colonic distension that results from a failed or inadequate pursestring suture, which can complicate the bottom-up first technique.

However, bottom-up first may confer some additional advantages: tumor visualization, location, and assessment are performed simultaneously; the resection margin is defined early in the operation; and an early decision can be made as to whether a coloanal anastomosis can be safely performed or if abdominoperineal resection will be necessary for complete tumor removal. There is also no forceful retraction, which might damage the mesorectum or fracture the tumor, and no possibility of blood or peritoneal fluid running down into the pelvic dissection.

Synchronous dissection has the obvious attraction of reducing the total operating time but does require 2 experienced surgeons and careful attention to the setup to allow both to operate efficiently and not hinder each others progress. Cooperation between 2 surgeons when joining the top and bottom surgical planes can improve exposure by retraction. The lower extremities have to be positioned in such a way as to permit both surgeons to operate comfortably without collisions. One of us (A.L.) has considerable experience of this approach with a mean operative time of 215 minutes. ${ }^{14}$

We believe that the transanal approach to tumors in the lower third of the rectum may make the mesorectal dissection both easier for the surgeon and safer for the patient, with both benefitting from the improved vision and accuracy of the dissection. The concern that there will be an increase in local recurrence when the dissection is started intersphincterically before insertion of the distal pursestring will be answered as soon as follow-up data of the early cases matures.

As experience with the technique develops, there is the potential for not only the distal mesorectal dissection to be performed, but also for dissection of the entire mesorectum, and even the vessel division and mobilization of the left colon and splenic flexure, without any laparoscopic abdominal surgery at all.

\section{REFERENCES}

1. Heald RJ, Moran BJ, Ryall RD, Sexton R, MacFarlane JK. Rectal cancer: the Basingstoke experience of total mesorectal excision, 1978-1997. Arch Surg. 1998;133:894-899.

2. Fleshman J, Sargent DJ, Green E, et al; Clinical Outcomes of Surgical Therapy Study Group. Laparoscopic colectomy for cancer is not inferior to open surgery based on 5-year data from the COST Study Group trial. Ann Surg. 2007;246:655-662.

3. Veldkamp R, Kuhry E, Hop WC, et al; COlon cancer Laparoscopic or Open Resection Study Group (COLOR). Laparoscopic surgery versus open surgery for colon cancer: short-term outcomes of a randomised trial. Lancet Oncol. 2005;6:477-484.

4. Guillou PJ, Quirke P, Thorpe H, et al; MRC CLASICC trial group. Short-term endpoints of conventional versus laparoscopic-assisted surgery in patients with colorectal cancer (MRC CLASICC trial): multicentre, randomised controlled trial. Lancet. 2005;365:1718-1726.

5. Ito M, Sugito M, Kobayashi A, Nishizawa Y, Tsunoda Y, Saito N. Relationship between multiple numbers of stapler firings during rectal division and anastomotic leakage after laparoscopic rectal resection. Int J Colorectal Dis. 2008;23:703-707.

6. Parks AG, Percy JP. Rectal carcinoma; restorative resection using a sutured colo-anal anastomosis. Int Surg. 1983;68:7-11.

7. Lyttle JA, Parks AG. Intersphincteric excision of the rectum. $\mathrm{Br}$ J Surg. 1977;64:413-416.

8. Parks AG, Nicholls RJ. Proctocolectomy without ileostomy for ulcerative colitis. Br Med J. 1978;2:85-88.

9. Marks J, Mizrahi B, Dalane S, Nweze I, Marks G. Laparoscopic transanal abdominal transanal resection with sphincter preservation for rectal cancer in the distal $3 \mathrm{~cm}$ of the rectum after neoadjuvant therapy. Surg Endosc. 2010;24:2700-2707.

10. Buess G, Kipfmüller K, Ibald R, et al. Clinical results of transanal endoscopic microsurgery. Surg Endosc. 1988;2:245-250.

11. Sylla P, Rattner DW, Delgado S, Lacy AM. NOTES transanal rectal cancer resection using transanal endoscopic microsurgery and laparoscopic assistance. Surg Endosc. 2010;24:1205-1210.

12. Rieder E, Spaun GO, Khajanchee YS, et al. A natural orifice transrectal approach for oncologic resection of the rectosigmoid: an experimental study and comparison with conventional laparoscopy. Surg Endosc. 2011;25:3357-3363.

13. Telem DA, Han KS, Kim MC, et al. Transanal rectosigmoid resection via natural orifice translumenal endoscopic surgery (NOTES) with total mesorectal excision in a large human cadaver series. Surg Endosc. 2013;27:74-80.

14. Fernández-Hevia M, Delgado $\mathrm{S}$, Castells A, et al. Transanal total mesorectal excision in rectal cancer: short-term outcomes in comparison with laparoscopic surgery. Ann Surg. 2015;261:221-227. 